\title{
CULTURA DE SEGURANÇA DO PACIENTE: AVALIAÇÃO PELOS PROFISSIONAIS DE ENFERMAGEM ${ }^{1}$
}

\author{
Daniele Bernardi da Costa ${ }^{2}$, Daniele Ramos ${ }^{3}$, Carmen Silvia Gabriel ${ }^{4}$, Andrea Bernardes ${ }^{5}$
}

${ }^{1}$ Artigo extraído da dissertação - Cultura de segurança do paciente sob a ótica da equipe de enfermagem em serviços hospitalares, apresentada ao Programa de Pós-Graduação em Enfermagem Fundamental da Escola de Enfermagem de Ribeirão Preto (EERP) da Universidade de São Paulo (USP), em 2014.

${ }^{2}$ Mestre em Enfermagem Fundamental. Docente da Universidade do Norte do Paraná. Londrina, Paraná, Brasil. E-mail: danielebernardi@hotmail.com

${ }^{3}$ Doutoranda do Programa de Pós-Graduação em Enfermagem da EERP/USP. Ribeirão Preto, São Paulo, Brasil. E-mail: danidsramos@yahoo.com.br

${ }^{4}$ Doutora em Enfermagem em Saúde Pública. Professora do Departamento de Enfermagem Geral e Especializada da EERP/USP. Ribeirão Preto, São Paulo, Brasil. E-mail: cgabriel@eerp.usp.br

${ }^{5}$ Doutora em Enfermagem Fundamental. Professora do Departamento de Enfermagem Geral e Especializada da EERP/USP. Ribeirão Preto, São Paulo, Brasil. E-mail: adreab@eerp.usp.br

\section{RESUMO}

Objetivo: avaliar a cultura de segurança do paciente das equipes de enfermagem no contexto hospitalar.

Método: sob o ponto de vista da enfermagem foi realizado um estudo quantitativo, tipo survey, transversal. A coleta de dados ocorreu entre junho e julho de 2013. Foi utilizado o questionário Hospital Survey on Patient Safety Culture, respondido por 437 profissionais da equipe de enfermagem, com taxa de resposta de $100 \%$.

Resultados: entre as doze dimensões avaliadas, apenas seis apresentaram taxas de respostas positivas maiores que $50 \%$, sendo frequência de relato de eventos $(61,4 \%)$, aprendizado organizacional - melhoria contínua (60,5\%), respostas não punitivas aos erros (58\%), trabalho em equipe dentro da unidade $(57,1 \%)$, passagem de plantão/turnos e transferências $(53,1 \%)$ e retorno da informação e comunicação sobre erro (51,8\%).

Conclusão: para o alcance de cultura de segurança positiva são necessárias ações de melhoria que envolvam a gestão e as chefias no que se refere: percepção geral da segurança; expectativas sobre o supervisor/chefe e ações promotoras da segurança; abertura da comunicação; apoio da gestão hospitalar para a segurança do paciente e trabalho em equipe entre as unidades.

DESCRITORES: Segurança do paciente. Enfermagem. Serviços hospitalares. Qualidade da assistência à saúde.

\section{PATIENT SAFETY CULTURE: EVALUATION BY NURSING PROFESSIONALS}

\footnotetext{
ABSTRACT

Objective: to evaluate the patient safety culture of nursing teams in the hospital context. Method: from a nursing point of view, a crosssectional quantitative study, of the survey type, was performed. The data collection occurred between June and July 2013. The questionnaire Hospital Survey on Patient Safety Culture was used, answered by 437 professionals of the nursing team, with a response rate of $100 \%$.

Results: among the twelve dimensions evaluated, only six had positive response rates greater than $50 \%$, being the frequency of event reporting $(61.4 \%)$, organizational learning - continuous improvement $(60.5 \%)$, non-punitive responses to errors (58\%), teamwork within the unit (57.1\%), shift/orderly and transfers (53.1\%), and feedback of information and communication about error (51.8\%).

Conclusion: in order to reach a positive safety culture, improvement actions involving management and leadership are required: general perception of safety; expectations about the supervisor/boss and actions that promote safety; the opening of communication; support of hospital management for patient safety and teamwork between units.
}

DESCRIPTORS: Patient Safety. Nursing. Hospital services. Quality of health care. 


\section{CULTURA DE SEGURIDAD PARA EL PACIENTE: EVALUACIÓN REALIZADA POR LOS PROFESIONALES DE ENFERMERÍA ${ }^{1}$}

\section{RESUMEN}

Objetivo: evaluar la cultura de seguridad del paciente de los equipos de enfermería en el contexto hospitalario.

Método: bajo el punto de vista de la enfermería fue realizado un estudio cuantitativo, tipo survey y transversal. La obtención de datos se realizó entre Junio y Julio del 2013. Se usó el cuestionario Hospital Survey on Patient Safety Culture que respondido por 437 profesionales del equipo de enfermería y con una taza de respuesta del $100 \%$.

Resultados: entre las doce dimensiones evaluadas apenas seis presentaron tazas de respuestas positivas mayores al 50\%, siendo ellas la frecuencia del relato de eventos (61,4\%), aprendizaje organizacional - mejora continua $(60,5 \%)$, respuestas no punitivas a los errores (58\%), trabajo en equipo dentro de la unidad $(57,1 \%)$, pasaje de guardia/turnos y transferencias $(53,1 \%)$ y proporcionar la información y comunicación sobre el error $(51,8 \%)$.

Conclusión: para llegar a la cultura de seguridad positiva son necesarias acciones de mejoramiento que incluyan la gestión y las jefaturas en lo que se refiere a la percepción general de la seguridad, expectativas sobre el supervisor/jefe y las acciones promotoras de la seguridad, abertura de la comunicación, apoyo de la gestión hospitalaria para la seguridad del paciente y el trabajo en equipo entre las unidades.

DESCRIPTORES: Seguridad del paciente. Enfermería. Servicios hospitalarios. Cualidad de la asistencia para la salud.

\section{INTRODUÇÃO}

Erros associados às instituições de saúde passaram a ser foco na mídia, com mais ênfase, após a publicação do relatório To err is human, do Institute of Medicine, em 1999, onde foram divulgados números altíssimos de mortes decorrentes de erros assistenciais e médicos. ${ }^{1}$ Mesmo com muitos esforços sendo realizados desde então, ainda assim, estudo publicado em 2013 estimou que mortes associadas a danos evitáveis acometam mais de 400.000 pessoas por ano e cerca de 4 a 8 milhões acometidos por danos graves, o que significa que diariamente ocorram, em média, 1.096 de mortes e 10.949 a 20.918 danos graves. ${ }^{2}$

A Joint Commission, em 2013, lançou suas primeiras metas de segurança do paciente para hospitais para o ano seguinte e atualmente estabeleceu as metas para o ano de 2016, sendo elas: melhorar a precisão da identificação do paciente, aprimorar a eficácia da comunicação entre os cuidadores, aperfeiçoar a segurança no uso de medicamentos, reduzir os danos associados aos sistemas de alarme clínicos, minimizar riscos de infecções associados ao cuidado e identificar os riscos de segurança inerentes aos pacientes pelos hospitais. ${ }^{3}$

No Brasil, também em 2013, instituiu-se, por meio da Portaria $n^{\circ} 529 / 13$, do Ministério da Saúde e a Resolução da Diretoria Colegiada (RDC) 36/2013, o Programa Nacional de Segurança do Paciente (PNSP), cujo objetivo é contribuir para a qualificação do cuidado em saúde em todos os estabelecimentos de saúde do território nacional. ${ }^{4}$ Somada a essa iniciativa, a RDC 36/2013, publicada pela Agência Nacional de Vigilância Sanitária (Anvisa), objetiva maior qualidade nos serviços de saúde através de ações de promoção de segurança e busca de melhoria contínua da qualidade. Juntos, representam a criação do Núcleo de Segurança do
Paciente (NSP) nos serviços de saúde através da execução do Plano de Segurança do Paciente em Serviços de Saúde (PSP). ${ }^{5}$

Dada a influência dos processos técnicos e organizacionais das instituições sobre os profissionais, rever os processos de trabalho é uma ferramenta essencial para o conhecimento dos problemas existentes e posterior implantação de barreiras para novos problemas relacionados à segurança. ${ }^{6}$

Cultura de segurança é o produto de valores individuais e de um grupo, atitudes, percepções, competências e padrão de comportamento que determinam o compromisso, o estilo e a proficiência da administração de uma organização saudável e segura. As instituições de saúde devem alertar-se para existência de cultura organizacional de baixas expectativas, caracterizada por falta ou falha de comunicação, mudanças ambíguas ou rápidas que não são comunicadas a todos os profissionais, troca de informações incompletas e a falta de informações. ${ }^{7}$

As instituições hospitalares têm incorporado ações em prol da cultura do paciente com o objetivo de oferecer assistência de excelência, diminuir custos e assegurar satisfação. Quando a segurança é instituída como processo cultural, constata-se maior consciência profissional quanto à cultura de segurança do paciente e ao compromisso ético do gerenciamento de risco para o profissional e para o paciente. ${ }^{8-9}$

$\mathrm{O}$ ambiente hospitalar deve ser seguro para que os profissionais possam relatar os erros ocorridos, conversar sobre eles, analisá-los junto às situações que os precederam, identificar os pontos frágeis dos processos a fim de repará-los, discutir estratégias de melhorias ${ }^{10}$ e priorizar a comunicação baseada na confiança entre os profissionais.

A avaliação da cultura de segurança do paciente permite reconhecer potencialidades e fragi- 
lidades que nortearão ações de melhorias de modo a construir uma cultura positiva e forte dentro das instituições de saúde. A equipe de enfermagem é o grande contingente de recursos humanos hospitalares e, na maioria das vezes, é responsável direta pela assistência, por isso, avaliar a cultura de segurança do paciente nessa população traz informações importantes e impactantes para as instituições hospitalares. Sendo assim, o objetivo deste estudo foi avaliar a cultura de segurança do paciente pela equipe de enfermagem no contexto hospitalar.

\section{MÉTODO}

Trata-se de um estudo descritivo, transversal, de abordagem quantitativa. Foi realizado em duas instituições hospitalares localizadas no norte do estado do Paraná, no Brasil. Quanto às características das instituições, a instituição A é um hospital universitário público geral e possui 316 leitos. A instituição B é um hospital geral privado de caráter filantrópico e possui 269 leitos.

A coleta de dados foi realizada na instituição A nos seguintes setores: Unidade de Internação Médico-Cirúrgica Masculina, Feminina, Moléstias Infecciosas, Centro Cirúrgico, Centro de Material Esterilizado, Unidades de Terapia Intensiva, Maternidade, Pediatria, Unidade de Terapia Intensiva Pediátrica, Unidade de Cuidados Intermediários Neonatal e Unidade de Terapia Intensiva Neonatal. Já os setores selecionados na instituição B foram: Unidades Clínicas, Unidades Cirúrgicas, Unidades de Terapia Intensiva, Pediatria, Maternidade, Unidade de Terapia Intensiva Neonatal e Pediátrica, Unidade de Cuidados Intermediários Neonatal, Centro Cirúrgico e Centro de Material Esterilizado.

Para o cálculo da amostra do estudo foi adotado nível de confiança de $95 \%$ e realizado segundo o cargo ocupado, totalizando 282 servidores entre auxiliares e técnicos de enfermagem e enfermeiros, 178 profissionais da instituição A e 104 profissionais da instituição B. Entretanto, a amostra final obteve participação efetiva de 437 servidores das instituições, pois houve um número maior de interessados do que o número calculado na amostragem. Apesar do cálculo amostral ter sido único para as duas instituições, o sorteio da amostragem foi realizado de forma independente es demais profissionais que demonstraram interesse em participar foram adicionados ao total sorteado. Cabe destacar que dos sorteados, todos os 282 servidores participaram da pesquisa.

Foram adotados os seguintes critérios de exclusão: profissionais com menos de seis meses na instituição; profissionais que estivessem, no período da coleta de dados, em férias, licenças ou afastamento do serviço; e estudantes de graduação e residência devido ao processo de formação e ausência de vínculo empregatício nas instituições. Como também foram excluídos os enfermeiros chefes das duas instituições devido a sua relação direta com as equipes de enfermagem, e não realização e acompanhamento da assistência de enfermagem de fato aos pacientes.

A coleta de dados ocorreu durante os meses de junho e julho de 2013, através da aplicação do questionário Hospital Survey on Patient Safety Culture (HSOPSC), desenvolvido e disponibilizado desde 2004 pela Agency for Health Research and Quality (AHRQ). O objetivo desse questionário é mensurar dimensões sobre a cultura de segurança, utilizado em diversos países do mundo e aborda as dimensões de cultura de segurança no âmbito individual, das unidades e também no âmbito hospitalar. O questionário foi traduzido e validado posteriormente para a língua portuguesa. ${ }^{11-12}$

O questionário é composto por nove seções que subdividem-se em 42 itens e aborda 12 dimensões, avaliadas pela média de cada item que a compõe, a saber: percepção geral da segurança; frequência de relato de eventos; expectativas sobre o supervisor/ chefe e ações promotoras da segurança; aprendizado organizacional - melhoria contínua; trabalho em equipe dentro das unidades; abertura da comunicação; respostas não punitivas aos erros; adequação de profissionais; retorno da informação e comunicação sobre erro; apoio da gestão hospitalar para a segurança do paciente; trabalho em equipe entre as unidades; e passagem de plantão e transferências.

Os itens são analisados através de escala Likert de cinco pontos, cujas categorias são de grau de concordância para as 12 dimensões. Cada dimensão foi avaliada segundo o percentual de respostas positivas, sendo o percentual obtido através do cálculo resultante da combinação das duas categorias mais altas de resposta; de cada dimensão as duas categorias mais baixas indicam resultados negativos referentes à cultura; e a categoria média demonstra neutralidade. ${ }^{11}$

As questões pertinentes ao número de eventos relatados e ao conceito dado à instituição em relação à segurança do paciente pelos servidores foram apresentadas com suas relativas frequências de respostas.

Quanto à avaliação das frequências obtidas nas dimensões, apesar dos autores do instrumento adotarem $75 \%$ de respostas positivas como corte para inter- 
pretar os achados como uma cultura de segurança do paciente positiva, consideraram esse corte arbitrário e indicaram que a instituição responsável pela aplicação e análise das respostas deve definir conforme suas necessidades. ${ }^{11}$ Assim sendo, ao ponderar a realidade das instituições hospitalares e do cenário brasileiro no que tange à temática de segurança do paciente, considerou-se cultura de segurança do paciente positiva a partir de $50 \%$ de respostas positivas.

A análise dos dados foi feita de forma descritiva através do programa estatístico Statistical Package for Social Sciences (SPSS), versão 17.0. O alfa de Cronbach total das dimensões foi 0,85.

O estudo foi avaliado e aprovado pelo Comitê de Ética em pesquisa da Escola de Enfermagem de Ribeirão Preto, Universidade de São Paulo, sob CAAE n ${ }^{\circ} 17533913.8 .0000 .5393$.

\section{RESULTADOS}

No que tange às características dos participantes e os dados profissionais, houve predominância do sexo feminino (84,7\%). Quanto ao item idade, observa-se que a maioria dos profissionais se encontra na faixa etária de 21 a 50 anos, somando $87 \%$. Em relação ao grau de escolaridade, a maior parte da população do estudo possui o ensino superior completo $(45,1 \%)$. No entanto, ao analisar a categoria profissional verifica-se a predominância de técnicos de enfermagem $(41,2 \%)$. Constata-se, ainda, que a maioria dos participantes atua nas unidades de internação clínica e cirúrgicas femininas e masculinas $(25,2 \%)$, seguida pela unidade de terapia intensiva $(18,3 \%)$ e pronto socorro $(10,8 \%)$, conforme Tabela 1 .

Dentre os participantes, em $94,7 \%$ dos cargos e funções exercidos, há interação ou contato direto com os pacientes.

Tabela 1 - Distribuição dos participantes segundo sexo, idade, cargo atual e tempo de trabalho na instituição. Londrina, PR, Brasil, 2013. (n=437)

\begin{tabular}{|c|c|c|c|}
\hline Variáveis & Categorias & $\mathrm{n}(\%)$ & Total N (\%) \\
\hline \multirow{2}{*}{ Sexo } & Feminino & $370(84,7 \%)$ & \multirow{2}{*}{$437(100 \%)$} \\
\hline & Masculino & $67(15,3 \%)$ & \\
\hline \multirow{5}{*}{ Idade } & $<20$ & $6(1,4 \%)$ & \multirow{5}{*}{$437(100 \%)$} \\
\hline & $21-30$ & $134(30,7 \%)$ & \\
\hline & $31-40$ & $114(26,1 \%)$ & \\
\hline & $41-50$ & $132(30,2 \%)$ & \\
\hline & $>51$ & $51(11,7 \%)$ & \\
\hline \multirow{3}{*}{ Cargo atual } & auxiliar de enfermagem & $151(34,6 \%)$ & \multirow{3}{*}{$437(100 \%)$} \\
\hline & técnico de enfermagem & $180(41,2 \%)$ & \\
\hline & enfermeiro (a) & $106(24,3 \%)$ & \\
\hline \multirow{8}{*}{ Tempo de trabalho na instituição } & $<1$ ano & $79(18,1 \%)$ & \multirow{8}{*}{$437(100 \%)$} \\
\hline & $1-5$ anos & $158(36,2 \%)$ & \\
\hline & $5-10$ anos & $44(10,1 \%)$ & \\
\hline & $10-15$ anos & $43(9,8 \%)$ & \\
\hline & $15-20$ anos & $52(11,9 \%)$ & \\
\hline & $20-25$ anos & $33(7,6 \%)$ & \\
\hline & $25-30$ anos & $14(3,2 \%)$ & \\
\hline & $>30$ anos & $14(3,2 \%)$ & \\
\hline
\end{tabular}

Os itens com piores avaliações nas dimensões, ou seja, com percentual de respostas positivas menores que $33,3 \%$, dizem respeito: a mudanças de plantão ou turno serem problemáticas para os pacientes (31,8\%); à quantidade de profissionais suficiente para atender a carga de trabalho $(16,9 \%)$; aos profissionais que consideram que seus erros, enganos ou falhas podem ser usados contra eles $(17,6 \%)$; aos supervisores que demandam que o trabalho seja realizado de forma mais rápida quando a pressão aumenta $(29,1 \%)$; ao supervisor/gerente não dá atenção suficiente aos problemas de segurança do paciente que acontecem repetidamente (20,4\%); ao fato de que a segurança do paciente jamais é comprometida em função da maior quantidade de trabalhos a ser concluída (32,5\%); aos profissionais sentirem-se à vontade para questionar as decisões ou ações dos seus superiores $(27,5 \%)$; aos profissionais que têm receio de perguntar quando algo não está correto (24,5\%); aos profissionais que são informados sobre mudanças implementadas a partir dos relatórios de eventos (23,3\%); e aos profissionais 
que se sentem à vontade para questionar as decisões ou ações dos seus superiores $(26,8 \%)$.

A Figura 1 mostra o percentual de respostas positivas em relação às doze dimensões avaliadas pelo instrumento. A dimensão com menos respostas positivas foi a Abertura da comunicação (36,6\%).

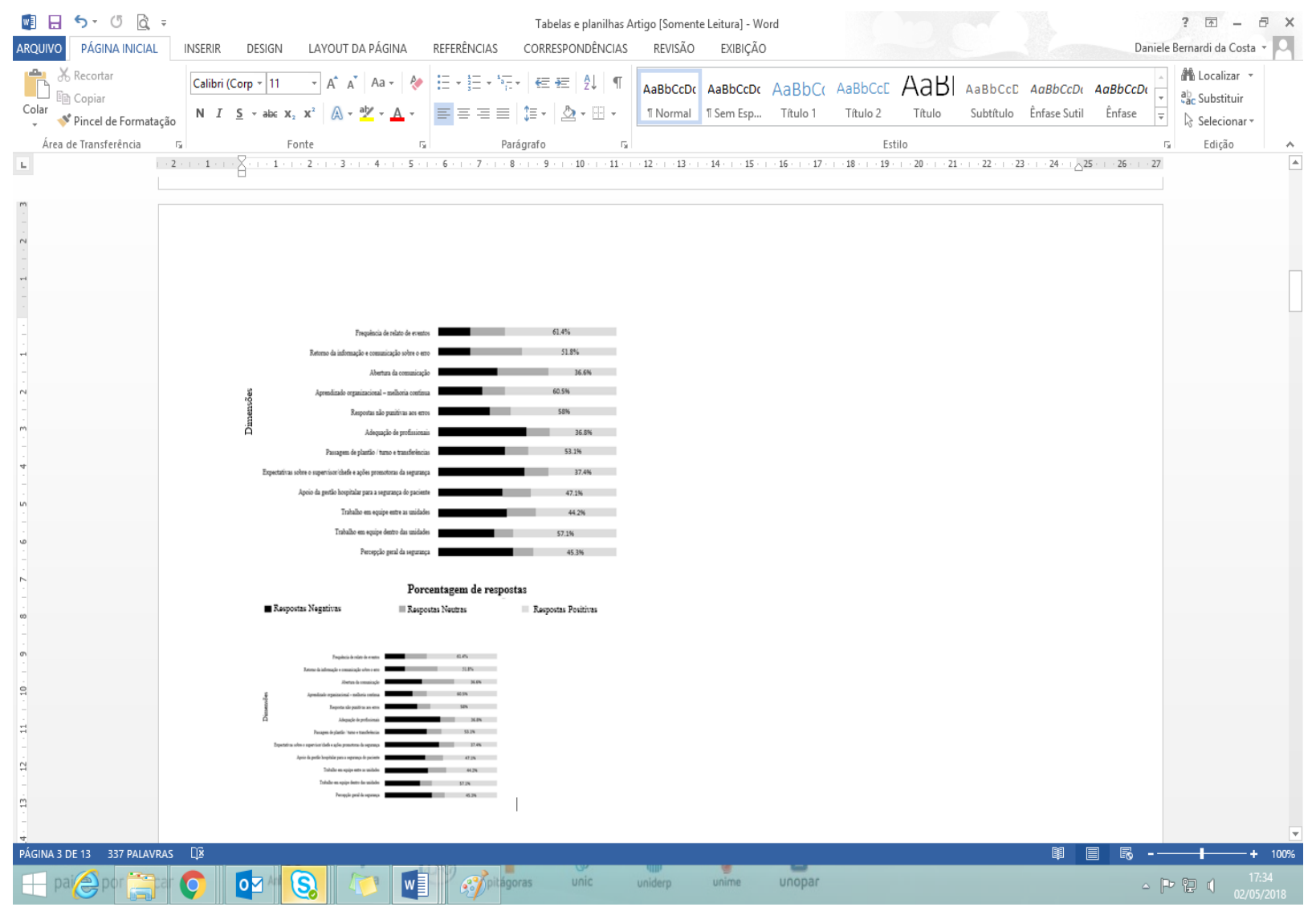

Figura 1 - Percentuais de escores positivos das dimensões de cultura de segurança do paciente de dois hospitais. Londrina, Brasil, PR, 2013

Na Figura 2, são apresentadas as frequências das respostas referentes ao conceito geral dado às instituições avaliadas relacionado à segurança do paciente. Pode-se inferir que a avaliação foi positiva, tendo em vista que a maior frequência de respostas se concentrou nas categorias excelente e muito boa, totalizando $(51 \%)$.

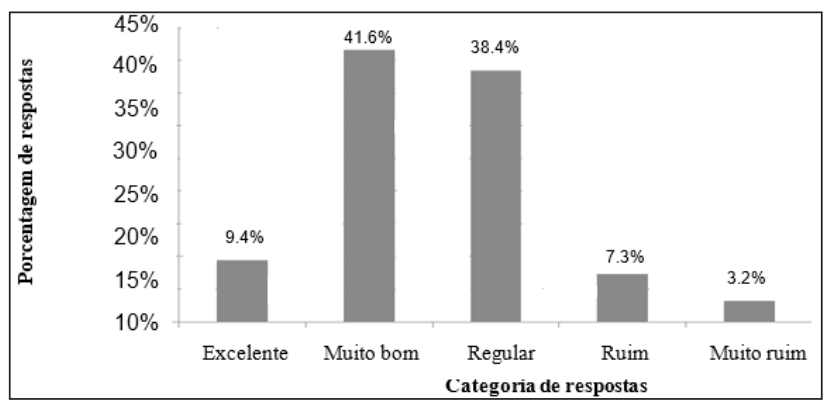

Figura 2 - Frequência dos conceitos gerais das instituições em relação à cultura de segurança do paciente de dois hospitais. Londrina, PR, Brasil, 2013
Nota-se que quando questionados sobre o número de notificações de eventos adversos preenchidos e apresentados nos últimos 12 meses, a grande maioria das respostas se concentrou entre 1 e 5 notificações, conforme apresentado na Figura 3.

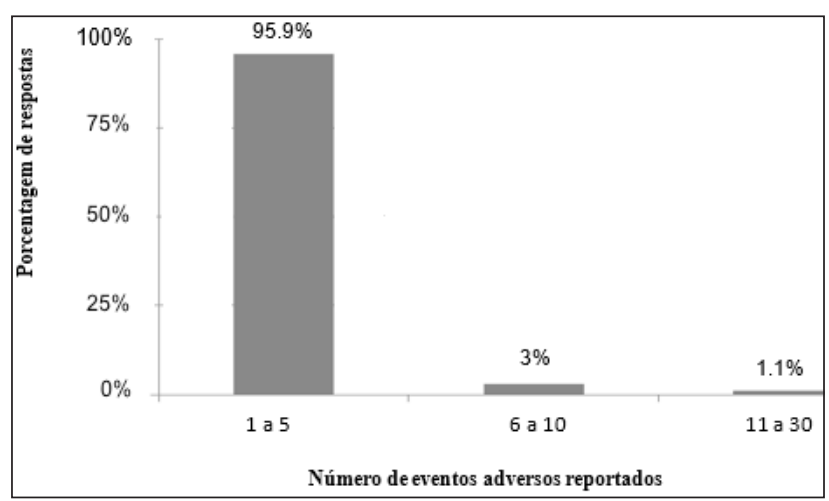

Figura 3 - Frequência de notificação de eventos adversos preenchidos e apresentados nos últimos 12 meses em dois hospitais. Londrina, PR, Brasil, 2013 


\section{DISCUSSÃO}

Os resultados demonstraram de maneira geral que a avaliação dos trabalhadores em relação à segurança do paciente das instituições não é positiva, sendo relevante destacar que problemas na segurança do paciente têm impactos financeiros, sociais e psicológicos importantes (aumento de custos e gastos em saúde, interrupção temporária ou permanente das atividades produtivas, dor, sofrimento emocional e impacto na taxa de mortalidade), que atingem tanto o paciente quanto a instituição de saúde. Por isso, a cultura de segurança do paciente tem papel fundamental para a definição de comportamentos e implementações de ações que visem à melhoria da qualidade institucional. É a partir do reconhecimento de potenciais e fragilidades institucionais e profissionais que surgem oportunidades de crescimento e resultados cada vez mais positivos, tanto para os pacientes quanto para as instituições de saúde. ${ }^{13}$

Dentre as dimensões do instrumento que apresentaram as avaliações mais negativas pelos profissionais, destacam-se tópicos relacionados à carga de trabalho; comunicação, cultura punitiva e hierarquizada e questões relacionadas a supervisão e liderança, apontadas em dimensões avaliadas negativamente tais como quantidade de profissionais insuficiente para dar conta da carga de trabalho, os profissionais consideram que seus erros, enganos ou falhas podem ser usados contra eles, o supervisor/ gerente não dá atenção suficiente aos problemas de segurança do paciente, os profissionais não se sentem à vontade para questionar as decisões ou ações dos seus superiores e não são informados sobre mudanças implementadas a partir dos relatórios de eventos. Ainda, ficou evidente o baixo número de notificações efetuadas pelos participantes.

Essas preocupações, bem como ações, em prol de melhorias devem expandir-se para todas as atividades, profissões e cargos dentro das instituições de saúde. Entretanto, sabe-se que as sobrecargas de trabalho nas equipes têm influência na qualidade das atividades profissionais.

Em especial, na equipe de enfermagem, a associação entre carga de trabalho e eventos adversos é significativa alta, impactando negativamente na segurança do paciente. ${ }^{14}$ Parte dos erros estão relacionados a processos complexos, dispositivos utilizados para realização da assistência, fragmentação do atendimento, falhas no processo de comunicação e falta de padronização dos procedimentos realizados, devendo o trabalho em equipe fornecer garantias contra a falibilidade humana, fortalecendo, portanto, a cultura de segurança. ${ }^{15}$
De acordo com pesquisa desenvolvida em unidades de internação das áreas clínicas e cirúrgicas de um hospital universitário do sul do Brasil, ${ }^{16}$ a sobrecarga de trabalho propicia a incidência de alguns eventos como: queda do leito, infecção associada ao cateter venoso central, o absenteísmo e a rotatividade.

O problema de sobrecarga na enfermagem está intimamente relacionado ao quantitativo insuficiente de profissionais. A relação existente entre a segurança do paciente e o quantitativo de enfermagem enfatiza a importância do correto dimensionamento de recursos humanos, uma vez que as taxas de mortalidade têm sido mais elevadas em hospitais cujo dimensionamento é inadequado. ${ }^{14}$

Nesse sentido, estudo realizado com o mesmo instrumento considerou o quantitativo reduzido de profissionais como um dos fatores para a subnotificação de eventos adversos ${ }^{17}$, o que, neste estudo, em conjunto com a sobrecarga totalizam uma taxa de $95,9 \%$ de números de eventos entre 1 e 5 , nos últimos 12 meses antecedentes à pesquisa.

A maioria dos servidores que responderam ao instrumento foram os técnicos de enfermagem. Estudo recente realizado em hospital público de média complexidade mostra que os técnicos de enfermagem são a maior categoria de trabalhadores dentro do ambiente hospitalar, totalizando 50,9\% dos trabalhadores. ${ }^{18}$ Tendo isso em vista, uma vez que a equipe de enfermagem é composta por enfermeiros, técnicos e auxiliares, definir o perfil dos trabalhadores pode indicar possíveis abordagens para a construção de uma cultura de segurança do paciente positiva ou fortalecimento da cultura já existente. Dessa forma, esta deve ser a categoria que mais precisa ser trabalhada no que tange a estratégias para melhoria da segurança do paciente, pois são eles os responsáveis pelos cuidados diretos aos pacientes no país.

Além disso, melhorias nos processos assistenciais gerenciados pelos enfermeiros e mudanças que beneficiam usuários dos sistemas, como também os trabalhadores, são potencializadas a partir de uma comunicação verdadeira, com respeito entre os profissionais e sustentada na interação e cooperação entre os saberes e fazeres profissionais. Uma das maneiras de melhorar a comunicação e o trabalho em equipe é enfatizar programas de treinamento em gestão de recursos humanos, com o objetivo de diminuir os níveis de autoridades, e realizar dinâmicas a fim de aumentar a interação entre os profissionais, tanto da mesma unidade como de unidades distintas. ${ }^{7-19}$ 
Além das ações que objetivem a segurança do paciente, há necessidade de que elas transcendam os profissionais da equipe de enfermagem e tornemse uma cultura da instituição. $\mathrm{O}$ envolvimento das equipes de gestão é fundamental para disseminar a cultura de segurança do paciente e, consequentemente planejar, implementar e avaliar diversas ações de melhoria.

Achados em relação à preocupação com segurança do paciente revelam que a administração não propicia clima de trabalho focado na segurança do paciente, muito menos demonstra interesse e iniciativas que a promovam. ${ }^{20}$

Por meio da notificação de eventos adversos, os profissionais conseguem enxergar os riscos presentes nos processos de trabalho, adotar medidas de prevenção dos eventos ou de barreiras à sua reincidência e também avaliar as medidas implementadas a fim de evidenciar melhorias na prática do trabalho. ${ }^{21}$ Os profissionais também devem ser constantemente orientados sobre a importância de tais registros e do preenchimento correto e completo, ao passo que os formulários de notificações devem ser claros, simples, de fácil compreensão e preenchimento. Ademais, soma-se a esses fatos a necessidade de compreensão acerca dos eventos adversos pelos profissionais envolvidos e a adoção de uma cultura não punitiva frente ao evento instalado. ${ }^{22-23}$

Na perspectiva de que a participação do profissional é fundamental para o desenvolvimento do cuidado de qualidade, o seu preparo está diretamente associado ao aperfeiçoamento dos sistemas e ao processo do cuidar, o qual deve ser focado no trabalho em equipe, no cuidado centrado no paciente, em práticas baseadas em evidências, no uso da informática e na melhoria da qualidade. ${ }^{24}$

A valorização da segurança do paciente e a implantação de medidas para essa finalidade devem ser prioridade de todos os profissionais, principalmente daqueles que ocupam cargos situados no topo do organograma, no intuito de que os resultados positivos sejam evidenciados nos processos de trabalho no âmbito da segurança do paciente. As instituições devem viabilizar meios que contribuam para análise dos seus processos de trabalho, sobretudo priorizar a segurança do paciente por meio de boas condições estruturais de trabalho aos profissionais e conhecimento dos problemas existentes a fim de minimizá-los e evitá-los quando possível.

No intuito de fortalecer a cultura de segurança do paciente, os gestores devem atentar-se a um modelo de gestão participativo, com estruturas mais horizontais e que atendam as necessidades dos pacientes e dos profissionais. Além disso, as lideranças das instituições de saúde devem coordenar a diversidade entre os profissionais de saúde e suas especialidades, de modo que o trabalho desenvolvido seja coordenado, eficaz e seguro para todos.

Deve-se considerar que o contínuo e intenso aprimoramento do serviço hospitalar só alcançará resultados efetivos se os profissionais de enfermagem estiverem comprometidos com a ruptura de um atendimento mecanizado e almejar um ambiente organizacional de excelência. Torna-se imperioso conhecer como estes trabalhadores, que representam a maior categoria profissional no contexto hospitalar, avaliam a segurança do paciente, por meio de um instrumento válido e confiável que possibilite um diagnóstico consistente das potencialidades e fragilidades entre o que é esperado - o ideal - e o que concretamente acontece - o real - na organização. Desta forma, fornecendo, subsídios para o desenvolvimento e consolidação de políticas institucionais de qualidade voltadas para a priorização das áreas mais frágeis com vistas à garantia de práticas seguras e qualificadas, assim como a valorização das pessoas envolvidas no processo de trabalho em saúde, sejam trabalhadores ou usuário.

\section{CONCLUSÃO}

Em relação à avaliação da cultura de segurança do paciente, o questionário adotado pode se tornar um importante aliado das instituições para reconhecer o momento cultural institucional. Os dados fornecidos através de sua aplicação auxiliam no diagnóstico da qualidade por ser a segurança do paciente uma importante dimensão da qualidade e também pelo fato de que a segurança do paciente influencia diretamente nas taxas de mortalidade, com impactos financeiros, sociais e psicológicos consideráveis.

O presente estudo revelou que as instituições devem repensar, junto aos seus profissionais, em especial a equipe de enfermagem, seus processos de notificação de eventos adversos por se constituir em uma ferramenta fundamental para promover a segurança do paciente. Ela fornece dados importantes nos processos de melhoria da qualidade, pressupondo análises e avaliações, implementação de barreiras, revisões de processos assistenciais e gerenciais, entre outras ações.

Algumas limitações podem ser apontadas na pesquisa, como a aplicação do questionário somente para a equipe de enfermagem. Como as instituições 
de saúde são compostas por diversos profissionais, a inclusão de diferentes categorias profissionais nas pesquisas pode fazer com que os resultados sejam mais fidedignos sobre a realidade da cultura de segurança do paciente nas instituições de saúde.

Conclui-se, por fim, que avançar nesse tema é desafiador para as instituições de saúde. Dessa forma, a partir da atual pesquisa acredita-se que outras possam ser desenvolvidas com o objetivo de conhecer e avaliar a cultura de segurança nas instituições de saúde, a fim de consolidar uma cultura de segurança construtiva. O estímulo à cultura de segurança do paciente e à implantação de metas que objetivem a prevenção de eventos adversos são imprescindíveis para a melhoria dos sistemas de saúde. Prevenir tais eventos é atualmente um grande desafio para o aprimoramento da qualidade na saúde.

\section{REFERÊNCIAS}

1. Institute of Medicine (US) Committee on Quality of Health Care in America; Kohn LT, Corrigan JM, Donaldson MS, editors. Washington (DC): National Academies Press (US); 2000.

2. James JT. A new, evidence-based estimate of patient harms associated with hospital care. J Patient Saf [Internet]. 2013 Sep [cited 2015 Oct 05]; 9(3):122-8. Available from: 10.1097/PTS.0b013e3182948a69.

3. The Joint Commission. National Patient Safety Goals Effective January 1, 2016 [Internet]. Chicago (IL): 2015 [cited 2015 Oct 05]. Available from: http://www. jointcommission.org/assets/1/6/2016_NPSG_HAP. pdf

4. Ministério da Saúde (BR). Portaria $N^{\circ} 529$ de 1 de abril de 2013: institui o Programa Nacional de Segurança do Paciente (PNSP) [Internet]. Available from: http:/ / bvsms.saude. gov.br/bvs/saudelegis/gm/2013/ prt0529_01_04_2013. html

5. Ministério da Saúde (BR). Agência Nacional de Vigilância Sanitária. Resolução da Diretoria Colegiada 36 de 25 de julho de 2013: Institui ações para a segurança do paciente em serviços de saúde e dá outras providências. Brasília (DF): MS; 2013.

6. Silva AEBC. Segurança do paciente: desafios para a prática e a investigação em Enfermagem. Rev Eletr Enf [Internet]. 2010 Sep [cited 2014 Jul 01]; 12(3):422. Available from: http://dx.doi.org/10.5216/ree. v12i3.11885

7. Watcher RM. Compreendendo a segurança do paciente. Porto Alegre (RS): Artmed; 2010.

8. Claro CM, Krocockz DVC, Toffolleto MC, Padilha KG. Adverse events at the intensive care unit: nurses' perception about the culture of nopunishment. Rev Esc Enferm USP [Internet]. 2011 Mar [cited2014 Jul 01]; 45(1):167-72. Available from: http:/ / www.scielo.br/scielo.php?script=sci_ arttext\&pid=S0080-62342011000100023

9. Oliveira RM, Leitão IMTA, Silva LMS, Figueiredo SV, Sampaio RL, Gondim MM. Strategies for promoting patient safety: from the identification of the risks to the evidence-based practices. Esc Anna Nery [Internet]. 2014 Mar[cited 2015 Jun 02]; 18(1):122-9. Available from: http://www.scielo.br/scielo.php?script=sci arttext\&pid=S1414-81452014000100122\&lng=en\&nr $\mathrm{m}=$ iso

10. Reis CT, Martins M, Laguardia J. A segurança do paciente como dimensão da qualidade do cuidado de saúde: um olhar sobre a literatura. Ciênc Saúde Coletiva [Internet]. 2013 [cited 2014 Jun 07]; 18(7):202936. Available from: http:/ / dx.doi.org/10.1590/S141381232013000700018

11. Agency for Healthcare Research and Quality. Hospital Survey on Patient Safety Culture. Rockville (US): Agency for Healthcare Research and Quality; 2004.

12. Reis CT, Laguardia J, Martins M. Adaptação transcultural da versão brasileira do Hospital Surveyon Patient Safety Culture: etapa inicial. Cad Saúde Pública [Internet]. 2012 Nov [cited 2015 Mar 02]; 28: 2199-210. Available from: http://www. scielo.br/scielo.php?script=sci_arttext\&pid=S0102311X2012001100019

13. National PatientSafety Foundation. Livre de danos: acelerar a melhoria da segurança do paciente - quinze anos depois de To Err Is Human [Internet]. Boston (US): The National Patient Safety Foundation. 2015. Available from: https://c.ymcdn.com/sites/npsf. site-ym.com/resource/resmgr/PDF/Free_from_ Harm_portugues-br.pdf

14. Rafferty AM, Clarke SP, Coles J, Ball D, James P, McKee M, et al. Outcomes of variation in hospital nurse staffing in English hospitals: cross-sectional analysis of survey data and discharge records. Int J Nurs Stud [Internet]. 2007 Feb [cited 2015 Jun 03]; 44(2):175-82. Available from: https:// www.ncbi.nlm. nih.gov/pmc/articles/PMC2894580/

15. Suresh GK. Measuring patient safety in neonatology. Am J Perinatol [Internet]. 2012 Jan [cited: 2015 Mar 03]; 29(1):19-26. Available from: https:/ / www.thiemeconnect.com/DOI/DOI?10.1055/s-0031-1286183

16. Conselho Federal de Enfermagem. Comissão de Business Intelligence. Análise de dados dos profissionais de enfermagem existentes nos Conselhos Regionais [Internet]. Departamento de Tecnologia da Informação: COFEN. 2011 [cited: 2014 Feb 10]. Available from: http://www.portalcofen.gov.br/ atlas/

17. Macedo TR, Rocha PK, Tomazoni A, Souza S, Anders JC, Davis K. The culture of patient safety from the perspective of the pediatric emergency nursing team. Rev Esc Enferm USP [Internet]. 2016 [cited 2016 Set 15]; 50(5):756-62. Available from: http://dx.doi. org/10.1590/S0080-623420160000600007 
18. Morais JAD, Silva JMTS, Haddad MDCFL, Cavalcante PPS. Processo de contratação coletiva de trabalhadores em hospital público de média complexidade. Rev Enferm Cent O Min [Internet]. 2015 [cited 2015 Set 15]; 4(2):1173-84. Available from: http://www.seer. ufsj.edu.br/index.php/recom/article/view/540

19. Santos JLG, Prochnow AG, Lima SBS, Leite JL, Erdmann AL. Communication conceptions in hospital nursing management between head nurses in a university hospital. Rev Esc Enferm USP [Internet]. 2011 Aug [cited 2015 Set 05]; 45(4):959-65. Available from: http://www.scielo. br $/$ scielo.php? script $=$ sci_arttext\&pid $=$ S008062342011000400024\&lng=en\&nrm=iso\&tlng=pt

20. Chaudhry B, Wang J, Wu S, Maglione M, Mojica W, Roth E. et al. Systematic review: Impact of health information technology in quality, efficiency, and costs of medical care. Ann Intern Med [Internet]. 2006 May [cited 2015 Out 15]; 144:742-52. Available from: http:/ / annals.org/article.aspx?articleid=723406

21. Carneiro FS, Bezzera ALQ, Silva AEBC, Souza LP,
Paranaguá TTB. Eventos adversos na clínica cirúrgica de um Hospital Universitário: instrumento de avaliação da qualidade. Rev Enferm UERJ [Internet]. 2011 [cited 2015 Nov 18]; 19(2):204-11. Available from: http://www.facenf.uerj.br/v19n2/v19n2a06.pdf

22. Bezerra ALQ, Silva AEBC, Branquinho NCSS, Paranaguá TTB. Análise de queixas técnicas e eventos adversos notificados em um hospital sentinela. Rev Enferm UERJ [Internet]. 2009 Out/Dez [cited 2015 Nov 20]; 17(4):467-72. Available from: http://www. facenf.uerj.br/v17n4/v17n4a02.pdf

23. Duarte SCM, Stipp MAC, Silva MM, Oliveira FT.Adverse events and safety in nursing care. Rev Bras Enferm [Internet]. 2015 [cited 2015 Ago 23]; 68(1):14454. Available from: http://dx.doi.org/10.1590/00347167.2015680120p

24. Rede Brasileira de Enfermagem e Segurança do Paciente. Estratégias para a segurança do paciente: manual para profissionais da saúde. Porto Alegre (RS): EDIPUCRS, 2013.
Correspondência: Daniele Bernardi da Costa

Rua Uruguai, 1500, ap 403

86010-210 - Centro, Londrina, PR, Brasil

E-mail: danielebernardi@hotmail.com
Recebido: 16 de setembro de 2016 Aprovado: 11 de outubro de 2017

This is an Open Access article distributed under the terms of the Creative Commons (CC BY). 